\title{
UTILIZAÇÃO DOS CONHECIMENTOS SOBRE COMUNICAÇÃO POR ALUNOS DE GRADUAÇÃO EM ENFERMAGEM
}

\author{
Maria Cristina Pinto de Jesus* \\ Mércia Heloísa Ferreira Cunha**
}

\begin{abstract}
JESUS, M.C.P.; CUNHA, M.H.F. Utilização dos conhecimentos sobre comunicação por alunos de graduação em
\end{abstract} enfermagem. Rev.latino-am.enfermagem, Ribeirão Preto, v. 6, n. 1, p. 15-25, janeiro 1998.

Este estudo descritivo teve como objetivo identificar as estratégias de comunicação terapêutica utilizadas pelos alunos das Escolas de Enfermagem da Universidade Federal de Minas Gerais e Universidade Federal de Juiz de Fora, durante suas atividades práticas. O método de coleta de dados constou de observações das interações dos alunos com pacientes hospitalizados e de entrevistas das pesquisadoras com os alunos. Constatou-se que, embora $90 \%$ dos alunos relatassem conhecer os conteúdos sobre comunicação, eles apresentaram dificuldades em desenvolver na prática os conhecimentos teóricos sobre comunicação.

UNITERMOS: comunicação, interação aluno-paciente; relacionamento terapêtico

\section{INTRODUÇÃO}

O indivíduo, em seu meio social, ou seja, em sua interação com outros seres humanos, utiliza a comunicação como veículo dessa interação. Entendendo o homem como ser social e membro de uma espécie, concorda-se com GARCIA \& AUGUSTO (1978) segundo os quais "as sociedades não poderiam existir sem contatos significativos entre pessoas", o que atribui à comunicação a importância de requisito básico para essa existência. Os autores acrescentam que pensamentos, crenças, esperanças e anseios podem, assim, ser direta ou indiretamente trocados no presente e durante um certo período de tempo; e que “...as idéias, os aspectos significativos dos símbolos transmitidos no ato social, constituem a essência da sociedade humana" (p.305).

A comunicação seria portanto uma condição sinequa-non da vida humana e da ordem social (WATZLAWICK et al.,1981). Nesse prisma cumpre caracterizar-se o processo de comunicação.

Para IRVING (1975), a comunicação pode ser definida como todos os passos dados, em uma relação em duplo sentido, entre a pessoa e seu meio ambiente. Coloca que a comunicação humana consiste especificamente na transmissão de uma mensagem que desencadeia uma resposta.

STEFANELLI (1983) considera a comunicação como condição fundamental da vida humana que abrangeria todos os procedimentos por meio dos quais um ser humano poderia afetar o outro.

Em seu estudo MENDES et al. (1988) concluem que a comunicação pode ser considerada como comportamento que objetiva a reflexão, o relacionamento, a troca de informações, de idéias, de imagens e de sentimentos visando ao atendimento humano e ao exercício da influência intencional.

Nos conceitos acima apresentados verificam-se elementos comuns. Percebe-se assim que a comunicação poderia ser descrita como um processo comportamental de troca ou inter-influência que se constitui em elemento básico de unidade social.

A prática de enfermagem envolve necessariamente uma relação interpessoal. Torna-se clara, portanto, a importância da comunicação como instrumento de enfermagem. Alguns autores consideram a comunicação como um instrumento básico, uma habilidade indispensável ao desempenho profissional e afirmam que é a comunicação que possibilita o relacionamento terapêutico enfermeiro-paciente (HORTA, 1971; STEFANELLI, 1990).

No sentido da humanização e personalização da assistência de enfermagem, a interação enfermeiropaciente deve ser efetiva uma vez que sem envolvimento não é possível uma percepção mais clara do outro.

\footnotetext{
* Enfermeira, professora adjunto no Departamento de Enfermagem Básica da Faculdade de Enfermagem da Universidade Federal de Juiz de Fora

** Enfermeira, professora adjunto no Departamento de Enfermagem Básica da Escola de Enfermagem da Universidade Federal de Minas Gerais
} 
MENDES et al. (1987) citam a afirmação de JOHNSON et al. de que "a comunicação habilidosa entre enfermeira e o paciente é vista por muitos como a essência da assistência de enfermagem" (p. 215).

É preciso, portanto, aprender a comunicar de forma efetiva e comunicar sempre, no sentido de promover interações gratificantes, pois "...qualquer que seja a situação, a comunicação só não melhora se você continuar não se comunicando". (ARANTES, 1983, p.105).

STEFANELLI $(1985,1990)$ elaborou uma lista de técnicas de comunicação terapêutica e de modos de comunicação não terapêutica, fundamentada em sua experiência e em estudos de diversos autores que se dedicaram a este tema, objetivando facilitar o estudo e a aplicação das mesmas para enfermeiros e estudantes de enfermagem. Segundo a autora, tais técnicas ou estratégias constituem-se em três grupos: o de expressão, que são estratégias que ajudam a descrição da experiência e estimulam a expressão verbal de pensamentos e sentimentos pelo paciente; as de clarificação, que são aquelas estratégias que ajudam a clarificar a comunicação verbal e as de validação, que são aquelas que ajudam a verificar se a compreensão da mensagem está correta (Anexo 1).

Ensinar aos estudantes de enfermagem as habilidades de comunicação de modo consistente e eficaz tem sido um problema para os educadores de enfermagem.

Conforme apontam FRIEDRICH et al. (1985), as habilidades de comunicação são ensinadas na escola de forma fragmentada, dando-se pouca atenção à prática, resultando daí a falta de competência do aluno entender e usar tais habilidades. As autoras apresentam o modelo de EGAN para ensinar as técnicas de comunicação que consistem na incorporação das técnicas orientadas para um objetivo, esquematizado em três fases a saber: a clarificação do problema, a identificação do foco para explorar o problema e a ação orientada ao objetivo.

Em seu estudo, FERRAZ (1991) afirma que existe uma incoerência entre o discurso e a prática de enfermagem, incluindo os docentes, já que os mesmos enfatizam mais o desenvolvimento da destreza manual do que os aspectos inerentes ao atendimento do homem como um todo, atendimento esse que requer dos alunos conhecimento de comunicação.

Para os alunos, principalmente os principiantes, pode se tornar uma tarefa difícil engajar-se na comunicação terapêutica ao mesmo tempo em que prestam cuidados ao paciente. No entanto, a comunicação, parte do papel da enfermagem, deve constituir uma técnica como qualquer outra. Portanto, os alunos devem praticar a técnica até que ela seja feita com destreza e bem feita (ATKINSON \& MURRAY,1989).
Em pesquisa junto aos alunos de enfermagem em campo clínico, FRIEDRICH et al. (1985) constataram que a gravação em fitas cassetes das interações alunopaciente possibilitou a identificação dos pontos fortes e fracos dos alunos quanto à comunicação, bem como a identificação dos problemas dos pacientes com vistas ao planejamento das estratégias de comunicação relevantes para as interações sucessivas.

Considerando a importância dos conhecimentos sobre comunicação para o ensino e a prática de enfermagem, de acordo com os autores citados anteriormente, temos nos preocupado ao exercer a nossa atividade como docentes em constatar que os alunos têm dificuldades para abordar o paciente, seja na explicação de rotinas, ao prestar informações sobre os procedimentos que executam ou simplesmente ouvi-lo.

Pretendeu-se nesse estudo responder à seguinte questão: os conhecimentos de comunicação obtidos pelos alunos de graduação em Enfermagem estão sendo utilizados nas atividades de interação com os pacientes?

\section{OBJETIVOS}

Identificar as estratégias de comunicação utilizadas pelos alunos de Enfermagem na interação com o paciente, nos campos de prática.

Analisar como o aluno percebe sua comunicação ocorrida na interação com o paciente.

\section{METODOLOGIA}

Optou-se por um estudo descritivo exploratório, realizado nas Escolas de Enfermagem da Universidade Federal de Minas Gerais (UFMG) e Universidade Federal de Juiz de Fora (UFJF), no período de setembro a novembro de 1994.

Participaram do estudo 10 alunos da Disciplina Fundamentos de Enfermagem e 10 alunos da Disciplina Enfermagem Médico-Cirúrgica, respectivamente, nos Cursos de Graduação em Enfermagem da UFMG e UFJF, que na ocasião estavam estagiando em enfermarias de clínica médica, clínica cirúrgica, unidades de oncologia e ambulatório. A coleta de dados foi realizada pelas próprias pesquisadoras, mediante a observação de interações aluno-paciente e entrevistas com os alunos. Utilizaram-se três instrumentos para o registro e análise dos dados: o formulário (segundo FERRAZ, 1991) para observação da interação (Anexo 2), o formulário para entrevista com os alunos (Anexo 3) e o instrumento para a apuração dos dados (Anexo 4). 
Optou-se por observar interações nas quais os alunos realizavam a entrevista para coleta de dados como uma estratégia para o planejamento da assistência de Enfermagem, considerando que esse é um momento da assistência em que é assegurada a comunicação face a face do aluno com o paciente.

Para a obtenção dos dados a respeito da percepção do aluno sobre a comunicação ocorrida na interação com o paciente utilizou-se o seguinte método: após o término das interações do aluno com o paciente as pesquisadoras e o aluno dirigiam-se a uma sala da unidade que proporcionasse condições para a entrevista. Nessa ocasião formulava-se a questão norteadora e registrava-se o mais fielmente possível o depoimento do aluno em impresso próprio (Anexo 3).

Os dados coletados foram organizados na forma de tabelas, que são analisadas e interpretadas a seguir.

\section{RESULTADOS E COMENTÁRIOS}

A população deste estudo constituiu-se de 20 alunos, sendo $16(80 \%)$ do sexo feminino e $4(20 \%)$ do sexo masculino.

Os dados obtidos na EE/UFMG e na Faculdade de Enfermagem da UFJF foram apresentados em conjunto, tendo em vista que não era objetivo do estudo comparar a comunicação aluno-paciente em ambas as escolas.

Para conhecer o conteúdo e a carga horária destinada ao ensino de comunicação em Enfermagem levantaram-se os dados através da análise dos programas e de conversas informais com docentes das disciplinas que ministram o conteúdo de comunicação nas instituições pesquisadas. Na EE/UFMG o conteúdo de comunicação é ministrado na disciplina Fundamentos de Enfermagem, perfazendo um total de 5 horas teóricas e na Faculdade de Enfermagem da UFJF o conteúdo de comunicação é desenvolvido nas Disciplinas: Fundamentos de Enfermagem I e Enfermagem em Saúde Mental, perfazendo um total de 15 horas teóricas.

Os dados obtidos mediante a observação das interações aluno-paciente são apresentados nas Tabelas de 1 a 4.

Tabela 1 - Frequência da comunicação terapêutica e modos de comunicação não terapêutica utilizada pelos alunos durante as entrevistas com os pacientes. Belo Horizonte - Juiz de Fora, 1994

\begin{tabular}{llll}
\hline & Comunicação & $\mathrm{N}^{0}$ & $\%$ \\
\hline Terapêutica & 463 & 47,4 \\
Não terapêutica & 513 & 52,6 \\
\hline Total & 976 & 100,0 \\
\hline
\end{tabular}

Partindo-se da análise dos diálogos e do contexto registrados segundo o formulário utilizado (Anexo 2) identificaram-se as estratégias de comunicação terapêutica e não terapêutica em cada interação do aluno com o paciente. Posteriormente, as pesquisadoras e uma especialista na área discutiram e validaram as identificações feitas. Houve concordância de aproximadamente $95 \%$ das identificações.

Pelos dados da Tabela 1 observou-se um total de 976 interações verbais classificadas segundo a listagem constante do Anexo 4. Das 976 interações dos alunos ocorridas nas entrevistas com os pacientes verificou-se que a frequência da comunicação terapêutica foi de 463 (47,4\%), enquanto que a da comunicação não terapêutica foi de 513 (52,6\%). Observa-se um quase equilíbrio na frequência de utilização das estratégias de comunicação terapêutica e não terapêutica, com leve predomínio de modos de comunicação não terapêuticos.

Comentando sobre o ensino de comunicação em enfermagem HARRISON et al. (1989) destacam que à medida que os alunos avançam no conhecimento e experiência, eles se sentem menos capazes de atuar terapeuticamente. Assim indagam: "os estudantes de enfermagem simplesmente esquecem as importantes habilidades terapêuticas, ou estão adotando estratégias mais pragmáticas para lidar com as contigências interpessoais e com o stress do ambiente de trabalho?" (p.89).

$\mathrm{Na}$ Tabela 2 apresentaram-se as estratégias de comunicação terapêutica utilizadas, mantendo-se as ordens em que foram listadas no Anexo 4. 
Tabela 2 - Frequência das estratégias de comunicação terapêutica utilizadas pelos alunos durante as entrevistas com os pacientes. Belo Horizonte - Juiz de Fora, 1994

\begin{tabular}{lcc}
\hline \multicolumn{1}{c}{ EXPRESSÃO } & $\mathrm{N}^{\circ}$ & $\%$ \\
\hline Fazerperguntas & 351 & 76,0 \\
Verbalizar interesse & 35 & 7,6 \\
Verbalizar aceitação & 20 & 4,3 \\
Repetir as últimas palavras ditas pelo paciente & 20 & 4,3 \\
Colocar em foco a idéia principal & 07 & 1,6 \\
Repetir comentários feitos pelo paciente & 06 & 1,3 \\
Fazer pergunta relativa aos dados comunicados & 05 & 1,1 \\
Devolver a pergunta feita pelo paciente & 03 & 0,6 \\
Estimular expressão de sentimentos subjacentes & 03 & 0,6 \\
Usar falas descritivas & 02 & 0,4 \\
Mantero paciente no mesmo assunto & 02 & 0,4 \\
Introduzir problema relacionado & 02 & 0,4 \\
Verbalizar dúvidas & 01 & 0,2 \\
CLARIFICAÇÃO & 03 & 0,6 \\
Descrever os eventos em seqüência lógica & & 0,4 \\
VALIDAÇÃO & 02 & 0,2 \\
Repetir a mensagem do paciente & 01 & 100,0 \\
Sumarizar o que foi dito na interação & 463 & \\
\hline Total & &
\end{tabular}

Observou-se na Tabela 2 que as estratégias mais utilizadas foram: "fazer perguntas" (76\%), "verbalizar interesse" (7,6\%), "verbalizar aceitação" $(4,3 \%)$ e "repetir as últimas palavras ditas pelo paciente" $(4,3 \%)$. Estas estratégias pertencem ao grupo das que STEFANELLI (1985) considera que auxiliam a descrição da experiência e a expressão de pensamentos e sentimentos do paciente.

FERRAZ(1991) encontrou resultados semelhantes, ou seja, a maior utilização das estratégias do grupo "expressão" ao analisar a comunicação enfermeiro-paciente em um hospital geral.

$\mathrm{Na}$ Tabela 3 podem-se visualizar as formas não verbais pelas quais os alunos comunicaram-se com os pacientes: mantendo-se próximo ao paciente, $(12,1 \%)$, expressando concordância, através da expressão facial, $(13,6 \%)$, emitindo afirmação com meneio da cabeça, $(11,4 \%)$, apresentando uma postura corporal de inclinação do tronco anterior, (12,1\%) e expressando-se verbalmente com tom de voz audível $(12,1 \%)$.

Tabela 3 - Sinais não verbais emitidos pelos alunos e registrados pelas pesquisadoras nas entrevistas com os pacientes. Belo Horizonte-Juiz de Fora/1994

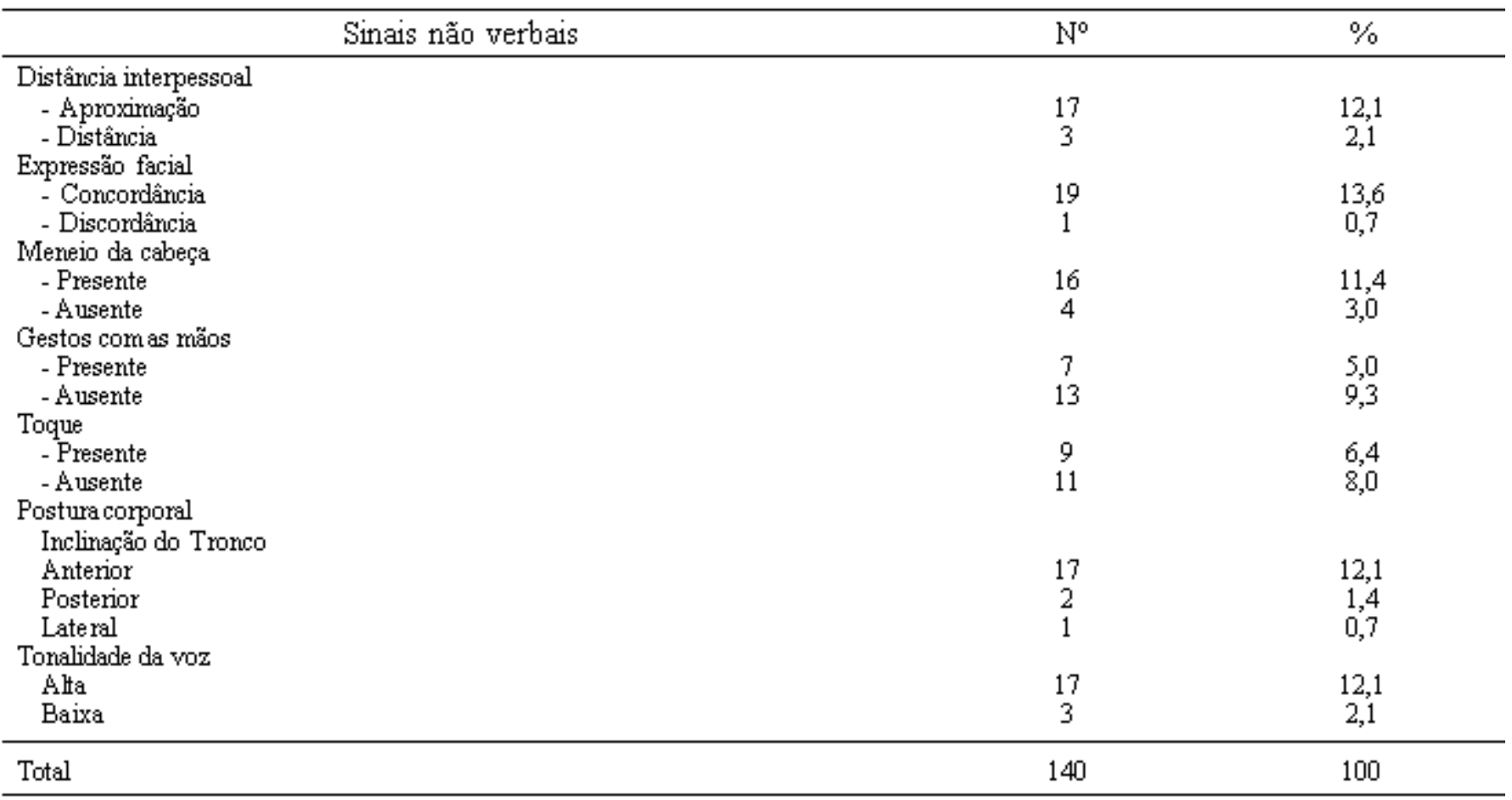


Observando-se os dados acima verifica-se que os alunos manifestaram explicitamente sinais não verbais de comunicação através da expressão facial, postura corporal e tonalidade de voz, dentre outros. De acordo com SILVA (1991), os nossos sentimentos estão expressos nos nossos atos, no nosso corpo, assim como os dos pacientes estão expressos nos deles. Frequentemente não falamos sobre nossos sentimentos mas os demonstramos de forma não verbal. Acredita-se que o aprendizado dos conteúdos sobre comunicação - a compreensão dos sinais não-verbais manifestos pelo paciente, tais como os expressos pelas ações e movimentos corporais, a postura corporal, os sinais vocais, o espaço entre os comunicadores, o toque e o momento em que as palavras são expressas - possa contribuir para que os alunos sejam capazes de interpretar não somente as mensagens verbais, mas também os sentimentos dos pacientes.

Analisando as interações observadas foi possível verificar que os modos de comunicação não terapêuticos foram utilizados pelos alunos, conforme os dados apresentados na Tabela 4.

Tabela 4 - Freqüência dos modos de comunicação não terapêutica, utilizada pelos alunos durante a entrevista com os pacientes. Belo Horizonte - Juiz de Fora, 1994

\begin{tabular}{lcc}
\hline \multicolumn{1}{c}{ Comunicação não terapêtuca } & $\mathrm{N}^{\circ}$ & $\%$ \\
\hline Induzir resposta & 253 & 49,3 \\
Fazer bateria de perguntas & 92 & 18,0 \\
Não saber ouvir & 90 & 17,5 \\
Mudar de assunto inadequadamente & 37 & 7,2 \\
Usar jargão profissional & 17 & 3,3 \\
Usar termos com conotação de julgamento & 09 & 1,7 \\
Oferecer falsa-tranqüilização & 04 & 0,8 \\
Menosprezar idéias do paciente & 04 & 0,8 \\
Dupla mensagem & 03 & 0,6 \\
Dar resposta estereotipada & 02 & 0,4 \\
Dar conselho & 01 & 0,2 \\
Comunicação unidirecional & 01 & 0,2 \\
\hline Total & 513 & 100,0 \\
\hline
\end{tabular}

"Induzir respostas", "fazer bateria de perguntas" e "não saber ouvir" foram os modos de comunicação não terapêuticos mais utilizados pelos alunos e obtiveram as frequências $49,3 \%, 18 \%$ e $17,5 \%$, respectivamente.

Os resultados obtidos demonstram que os alunos enfrentam dificuldades nos seus primeiros contatos com os pacientes. Tais dificuldades também foram encontradas por CANCHE et al. (1988), ao estudarem a comunicação aluno-paciente. Os autores apontam como dificuldade do aluno à obtenção de colaboração do paciente: a transposição de barreiras, a superação do medo, a apreensão, a ansiedade e a insegurança do aluno iniciante nas atividades do ciclo profissional do curso de Enfermagem.

A compreensão dos alunos sobre a sua própria comunicação com os pacientes foi obtida mediante a análise das entrevistas com os pesquisadores.

A análise dos depoimentos revelou que os alunos relataram dificuldades na comunicação com os pacientes, insegurança e inexperiência no contato com o mesmo. Pode-se citar como exemplo de suas falas: "acho que falta muito, porque faço pouco"; " a minha interação com o paciente está bem fraca. Ainda me sinto bem insegura"; "acho dificil me comunicar com o paciente"; "a gente tem mais medo de abordar o paciente do que ele em responder". Os alunos, em sua maioria, 90\%, afirmaram conhecer os conteúdos sobre comunicação, sendo as falas mais citadas: comunicar é "saber ouvir"; "utilizar linguagem adequada"; mostrar interesse pelo paciente"; "ouvir mais do que falar".

Os alunos mencionaram os conceitos de comunicação terapêutica e relacionamento terapêutico e reconheceram a importância da comunicação como um instrumento essencial para o planejamento da assistência de enfermagem, predominando as seguintes falas: " $a$ comunicação terapêutica é importantíssima, pois proporciona um levantamento das necessidades do paciente". "Acho que é muito válida, através da comunicação estabelecemos o diagnóstico de enfermagem"; "sem a comunicação eu não saberia as coisas que acontecem com o paciente".

Esses resultados apontam a percepção que os alunos têm das suas dificuldades na prática. Tal fato corrobora o questionamento das pesquisadoras de que o 
ensino das habilidades de comunicação continua centrado na teoria, uma vez que a maioria dos alunos tem domínio dos conteúdos teóricos mas não os aplica, verbalizando dificuldades em usar tais habilidades ao relacionar-se com o paciente. Por outro lado, acredita-se que, ao refletir sobre suas dificuldades na interação com o paciente, o aluno estará dando um primeiro passo para conscientizarse sobre as formas de comunicação que está utilizando ao desenvolver a prática da enfermagem.

\section{CONSIDERAÇÕES FINAIS}

Considera-se, após a análise dos dados deste estudo, que houve um quase equilíbrio na frequência de utilização das estratégias de comunicação terapêutica e não terapêutica, com leve predomínio dos modos de comunicação não terapêuticos.

As estratégias de comunicação terapêutica do grupo "expressão" foram as mais utilizadas pelos alunos e dentre os modos de comunicação não terapêuticos destacaram-se a indução de respostas, fazer baterias de perguntas e o não saber ouvir.

Cumpre ressaltar que os alunos manifestaram explicitamente sinais não verbais tais como: expressão facial de concordância, manter-se próximo ao paciente, emissão de concordância através do meneio da cabeça, postura de inclinação do tronco e expressão verbal com tom de voz audível.

Através da análise de depoimentos verificou-se que, embora $90 \%$ dos alunos relatassem conhecer o conteúdo de comunicação, os mesmos manifestaram dificuldades, insegurança e inexperiência na interação com os pacientes. Assim, acredita-se que embora o conteúdo de comunicação terapêutica seja trabalhado nas escolas de Enfermagem, a complexidade do processo de comunicação humana exige do aluno um tempo para o desenvolvimento das habilidades de comunicação.

Destaca-se por fim o fato de que o aluno necessita conscientizar-se da importância de desenvolver sua habilidade de comunicação terapêutica, estimulando o paciente a verbalizar os seus sentimentos.

Parece oportuno sugerir aos professores de enfermagem a implementação de ações que visem diminuir as dificuldades dos alunos no relacionamento com o paciente, tais como oferecer-lhes a oportunidade para o desenvolvimento das habilidades de comunicação antes que ingressem no ambiente hospitalar, assim como o incentivo à prática dos conceitos teóricos de comunicação sob supervisão dos docentes durante as atividades de estágio curricular.

\section{UTILIZATION OF COMMUNICATION KNOWLEDGE BY NURSING UNDERGRADUATE STUDENTS}

This descriptive study had as main purpose to identify therapeutic communication strategies used by Nursing students from the Nursing School of UFMG and the Nursing School of UFJF, during their practice.The method used for data collection consisted of observation on the interaction between students and inpatients and of interviews with the students. The results showed that although $90 \%$ of the students said they learned about communication, they had difficulties to practice in the field what they learned as theoretical knowledge.

KEY WORDS: communication, student-patient interaction, therapeutic relationship

\section{UTILIZACIÓN DE LOS CONOCIMIENTOS SOBRE COMUNICACIÓN POR LOS ALUMNOS DE PREGRADO EN ENFERMERÍA}

Este estudio descriptivo tuvo como objetivo analizar el conocimiento de las estrategias de comunicación terapéutica empleadas por los alumnos de las Escuelas de Enfermería de la Universidad Federal de Minas Gerais y Universidad Federal de Juiz de Fora, durante sus actividades prácticas. En la metodologia fueron realizadas observaciones de las interacciones. Además se entrevistaron 20 alumnos. En las observaciones fueron consideradas las interacciones de los alumnos con los pacientes hospitalizados y la entrevista de los investigadores con los alumnos. Fue constatado que aunque $90 \%$ de los alumnos relatoron conocer los contenidos sobre comunicación, ellos presentaron dificuldades en desarrollar en la práctica los conocimientos teóricos sobre comunicación.

TÉRMINOS CLAVES: comunicación, interacción alumno-paciente, relacionamiento terapéutico 


\section{REFERENCIAS BIBLIOGRÁFICAS}

01. ARANTES, D.V. As enfermeiras e a comunicação. Rev. Bras. Enfermagem, v.3, n. 3, p. 105, 1983

02. ATKINSON, L.D.; MURRAY, M.E. Fundamentos de enfermagem: introdução ao processo de enfermagem. Rio de Janeiro: Guanabara Koogan, 1989.

03. CANCHE, R.F. et al. A comunicação aluno/paciente segundo a percepção de graduandos de enfermagem. In: SIMPÓSIO BRASILEIRO DE COMUNICAÇÃO EM ENFERMAGEM, Ribeirão Preto, 1988. Anais. Ribeirão Preto: Escola de Enfermagem de Ribeirão Preto da USP, 1988. p. 175-186.

04. FERRAZ, A.F. Análise da comunicação enfermeiro paciente em hospital geral. São Paulo, 1991. p.106. Dissertação (Mestrado) Escola de Enfermagem, Universidade de São Paulo.

05. FRIEDRICH, R.M. et al. Teaching communication skills in an integrated curriculum. J. Nurs. Education, v. 24, n. 4, p. 164-166, 1985.

06. GARCIA, T.J.M.; AUGUSTO, D. Aplicação do processo de comunicação pela enfermagem obstétrica. Enfermagem Novas Dimensões, v. 4, n. 6, p. 303-307, 1978.
07. HARRISON, T.M. et al. Assessing nurses'communication: a cross-sectional study. Western J. Nurs.Res. v.11, n.1, p.7591,1989.

08. HORTA, W. de A. et al. O ensino dos instrumentos básicos da enfermagem. Rev. Esc.Enfermagem USP, v. 5, n. 1, p. 7-11, 1971.

09. IRVING, S. Comunicación e relaciones humanas. In: Enfermagem Psiquiátrica. México: Interamericana, 1975. p. 23-31.

10. MENDES, I.A.C. et al. Definições teórica e operacional do conceito de comunicação. Rev.Gaúch.Enfermagem, v. 8, n. 2, p.204-219, 1987.

11. MENDES, I.A.C. et al. O padrão de comunicação do enfermeiro com o paciente. Rev.Paul. Enfermagem, v. 8, n.1, p.13-15, 1988.

12. SILVA, M.J.P. da. Percebendo os sentimentos de maneira não verbal Rev.Paul.Enfermagem, v.10, n.3, p.128-32, set./dez. 1991.

13. STEFANELLI, M.C. Comunicação terapêutica enfermeira-paciente: avaliação do ensino. Rev.Enfermagem Científica, n.1, p.4-10, 1990.

14. _. A comunicação terapêutica. Rev. Paul. Enfermagem, v. 3, n. 3, p. 103-4, 1983.

$15 . \quad$ Ensino de técnicas e comunicação terapêutica enfermeira-paciente. São Paulo, 1985.163p. Tese (Doutorado)-Escola de Enfermagem, Universidade de São Paulo.

16. WATZLAWICK, P. et al. Pragmática da comunicação humana. São Paulo: Cultrix, 1981. 
ANEXO 1

Estratégias de comunicação terapêutica e da comunicação não terapêutica, segundo STEFANELLI (1985)

Estratégias de comunicação terapêutica

\section{EXPRESSÃO}

01. Permanecer em silêncio (uso terapêutico do silêncio)

02. Ouvir reflexivamente (saber ouvir)

03. Verbalizar aceitação

04 . Verbalizar interesse

05. Usar frases incompletas

06. Repetir comentários feitos pelo paciente

07. Repetir as últimas palavras ditas pelo paciente

08. Fazer perguntas

09. Fazer pergunta relativa aos dados comunicados

10. Introduzir problema relacionado

11. Devolver a pergunta feita pelo paciente

12. Usar frases descritivas

13. Manter o paciente no mesmo assunto

14. Permitir ao paciente que escolha o assunto

15. Colocar em foco a idéia principal

16. Verbalizar dúvidas

17. Dizer não

18. Estimular expressão de sentimentos subjacentes

\section{CLARIFICAÇÃO}

19. Estimular comparações

20. Solicitar que esclareça termos incomuns

21. Solicitar que precise o agente da ação

22. Descrever os eventos em seqüência lógica

VALIDAÇÃO

23. Repetir a mensagem do paciente

24. Pedir ao paciente para repetir o que foi dito

25. Sumarizar o que foi dito na interação

Comunicação não terapêutica

\footnotetext{
01. Não saber ouvir

02. Dar conselho

03. Ficar na defensiva

04. Oferecer falsa tranqüilização

05. Usar termos com conotação de julgamento

06. Colocar o paciente à prova

07. Dar resposta estereotipada

08. Menosprezar idéias do paciente

09. Desconfirmação

10. Dupla mensagem

11. Comunicação unidirecional

12. Induzir resposta

13. Não individualizar a comunicação

14. Usar jargão profissional

15. Fazer bateria de perguntas

16. Mudar de assunto inadequadamente
} 


\section{ANEXO 2}

Dados sobre a interação

Hospital:

Iniciais do Aluno:

Período no Curso de Graduação em Enfermagem:

Disciplina que está cursando:

Parte I: Guia para observação da interação

- abordagem inicial

- diálogos mantidos

- contexto em que ocorreu a interação

- comunicação não verbal ocorrida

Parte II: Registro da interação
Ala:

Sexo:

\begin{tabular}{|c|c|c|}
\hline Inicio: & Término: & Duração \\
\hline Comunicação do aluno & Comunicação do paciente & Observações \\
\hline & & \\
\hline & & \\
\hline & & \\
\hline & & \\
\hline & & \\
\hline & & \\
\hline & & \\
\hline
\end{tabular}




\section{ANEXO 3}

Formulário para entrevista

Hospital:

Inicias do Aluno:

Período no Curso de Graduação em Enfermagem:

Disciplina que está cursando:

Carga Horária:

$$
\begin{aligned}
& \mathrm{T}= \\
& \mathrm{P}=
\end{aligned}
$$

Fale sobre a comunicação ocorrida entre você e o paciente.
Ala:

Sexo: 


\section{ANEXO 4}

Instrumento para apuração dos dados

Hospital:

Ala:

Iniciais do Aluno:

Sexo:

Indicação das estratégias de comunicação terapêutica e da comunicação não terapêutica utilizada pelo aluno durante a interação com o paciente

1 - Estratégias de comunicação terapêuticas

\begin{tabular}{|c|c|c|}
\hline EXPRESSÄO & CLARIFICAÇÃO & VALIDAÇÃO \\
\hline $\begin{array}{l}\text { 01. Permanecer em silêncio } \\
\text { 02. Ouvir reflexivamente } \\
\text { 03. Verbalizar aceitação } \\
\text { 04. Verbalizar interesse } \\
\text { 05. Usar frases incompletas } \\
\text { 06. Repetir comentários feitos pelo } \\
\text { paciente } \\
\text { 07. Repetir as últimas palavras ditas pelo } \\
\text { paciente } \\
\text { 08. Fazer perguntas } \\
\text { 09. Fazer pergunta relativa aos dados } \\
\text { comunicados } \\
\text { 10. Introduzir problema relacionado } \\
\text { 11. Devolver a pergunta feita pelo } \\
\text { paciente } \\
\text { 12. Usar frases descritivas } \\
\text { 13. Manter o paciente no mesmo assunto } \\
\text { 14. Permitir ao paciente que escolha o } \\
\text { assunto } \\
\text { 15. Colocar em foco a idéia principal } \\
\text { 16. Verbalizar dúvidas } \\
\text { 17. Dizer "não" } \\
\text { 18. Estimular expressão de sentimentos } \\
\text { subjacentes }\end{array}$ & $\begin{array}{l}\text { 19. Estimular comparações } \\
\text { 20. Solicitar que esclareça termos } \\
\text { incomuns } \\
\text { 21. Solicitar que precise o agente } \\
\text { daação } \\
\text { 22. Descrever os eventos em } \\
\text { sequência lógica }\end{array}$ & $\begin{array}{l}\text { 23. Repetir a mensagem do } \\
\text { paciente } \\
\text { 24. Pedir ao paciente para repetir } \\
\text { o que foi dito } \\
\text { 25. Sumarizar o que foi dito na } \\
\text { interação }\end{array}$ \\
\hline
\end{tabular}

2 - Comunicação não terapêutica

01. Não saber ouvir

02. Dar conselho

03. Ficar na defensiva

04. Oferecer falsa tranqüilização

05. Usar termos com conotação de julgamento

06. Colocar o paciente à prova

07. Dar resposta estereotipada

08. Menosprezar as idéias do paciente

09. Desconfirmação

10. Dupla mensagem

11. Comunicação unidirecional

12. Induzir resposta

13. Não individualizar a comunicação

14. Usar jargão profissional

15. Fazer bateria de perguntas

16. Mudar de assunto inadequadamente 